\title{
Carrier trapping study on a Ge nanocrystal by two-pass lift mode electrostatic force microscopy
}

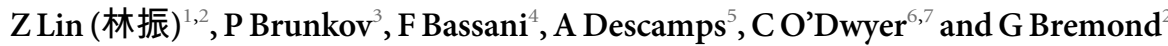 \\ 1 Institut des Nanotechnologies de Lyon-INL/UMR 5270, CNRS, Ecole Centrale de Lyon, 36 avenue Guy de Collongue, 69134 Ecully \\ Cedex, France \\ 2 Université de Lyon, Institut National des Sciences Appliquées de Lyon, Bât. Blaise Pascal, 20, avenue Albert Einstein—69621 Villeurbanne \\ Cedex, France \\ 3 Ioffe Physical-Technical Institute of the Russian Academy of Sciences, Saint-Pétersbourg, 194021, Russia \\ 4 Institut Matériaux Microélectronique Nanosciences de Provence, UMR CNRS 6242, Université Paul Cézanne, Avenue Escadrille \\ Normandie-Niemen-Case 142, F-13397 Marseille Cedex 20, France \\ 5 Centre Lyonnais de Microscopie, CLYM platform facility, 69621 Villeurbanne Cedex, France \\ 6 Department of Chemistry, University College Cork, Cork, Ireland \\ 7 Micro \& Nanoelectronics Centre, Tyndall National Institute, Lee Maltings, Cork, Ireland \\ E-mail: zhen.lin@univ-montp2.fr
}

Keywords: nanostructure, germanium nanocrystal, electrostatic force microscopy, memory device

\begin{abstract}
Trapped charges inside an isolated germanium nanocrystal ( $\mathrm{Ge} \mathrm{NC}$ ) have been studied by two-pass lift mode electrostatic force microscopy (EFM) measurements at room temperature. From visualized EFM images, electrons and holes were proven to be successfully injected and trapped in the Ge NC and distributed homogenously at the edge of its truncated spherical morphology. The Ge NC is found to have iso-potential surface and behave as a conductive material after being charged. It is also shown that the dominant charge decay mechanism during discharging of Ge NCs is related to the leakage of these trapped charges. A truncated capacitor model is used to approximate the real capacitance between the tip and Ge NC surface and to quantitatively study these trapped charges. These investigations demonstrate the potential for Ge nanocrystal memory applications.
\end{abstract}

Nowadays, semiconductor technology is facing a great challenge to improve device performance while reducing feature dimensions, approaching size regimes where surface effects become very important for electrical transport and biasing. This size downscaling in the microelectronics industry has initiated an explosive development of various microscopy techniques to probe and reveal new physical characteristics at the nanoscale such as Coulomb blockade, quantized charging effects [1-3] and single-electron transistors [4,5].

Due to the increasing demands for information storage and the significant scaling limitations of traditional memories, the non-volatile memory device composed of silicon ( $\mathrm{Si}$ ) or Ge nanodots is very promising [6-8]. Altering the oxide thickness and voltage operation can directly influence charge storage, and nanoscale crystals are promising since they have fast tunnelling-mediated write times $[9,10]$. Thus, the characterization and understanding of the charging mechanism in such nanostructures is of prime importance. However, most of the studies of charge traps in Ge nanocrystals were done by microscopy at micron length scales.

Variations on atomic force microscopy can provide simultaneous topography and various physical feature images with some additional applications such as scanning capacitance microscope (SCM), electrostatic force microscope (EFM), scanning tunnelling microscope (TUNA) and Kelvin probe force microscope (KPFM) [1116]. Electrostatic force microscopy (EFM) is used especially for characterizing materials for accurate local and non-destructive electrical properties for a wide range of characterizations such as surface potential, charge distributions and dielectric constant [17]. EFM is also able to easily and non-destructively inject and detect the localized charge in nanostructures, on or below the surface by a special two-pass lift mode. This ability has been used to study the distribution of trapped charges in silicon dioxide layer or implanted nano crystals [18$21,25,26]$. However, the characterisation of an isolated Ge NC was rarely studied by EFM at room temperature. 
Such nanostructures are of a great interest because the injected carriers are strongly constrained in their propagation, and interact with a finite geometry which should be generally the case in nano-electronic devices.

In this study, an isolated Ge NC on a silicon dioxide layer on $\mathrm{n}^{+}$type doped silicon (001) substrate has been shown to exhibit charge storage memory effects by EFM two-pass lift mode measurement at room temperature. The accurate phase signal conducted by electrostatic force interactions was used to determine the charge retention time inside the Ge NCs. The charge storage and retention effects are discussed in the context of Ge NC memory application. In order to quantitatively study these trapped charges, a truncated capacitor model was used to approximate the real capacitance between the tip and island surface. The quantity of charged electrons was calculated by analytical expression of the charge quantity as a function of the EFM phase signal.

These nano-scale Ge NCs have been fabricated on top of a very thin silicon dioxide layer using a dewetting processing. A $15 \mathrm{~nm}$ thick Ge layer was deposited by molecular beam epitaxy (MBE) over the $\mathrm{SiO}_{2}$ layer $5 \mathrm{~nm}$ in thickness at ambient temperature and was thermally annealed at $750{ }^{\circ} \mathrm{C}$ for 20 min under ultrahigh vacuum. This process leads to the formation of crystalline Ge NCs having an average diameter $\sim 150 \mathrm{~nm}$. As has been shown in previous work [22], the Ge NC diameter is $\sim 7$ times the nominal thickness of the Ge layer. Over the dots there is a natural oxide layer of $\sim 2 \mathrm{~nm}$ so that the sample surface is uneven, comprising a dispersion of Ge NCs, seen in figure 1.

In order to minimize the influence of morphology over the sample surface during charge measurement, electrostatic force microscopy (EFM) was chosen for charging experiment. EFM measurements are acquired in a two-pass lift mode. In this measurement, there is a constant separation between the tip and local surface topography [22] when the tip rises to the lift scan height, and this maintains constant van der Waal's interaction. This allows the imaging of relatively weak but long-range electrostatic interactions while minimizing the influence of topography.

Modelled by simple parallel plate capacitor, the electrostatic force between a conductive tip and a sample surface can be described as:

$$
F=\frac{1}{2} \frac{\partial C}{\partial z}(\Delta V)^{2}=\frac{1}{2} \frac{\partial C}{\partial z}\left(V_{t}-V_{s}\right)^{2}
$$

where $C$ is the effective capacitance between the EFM probe and the sample; $V_{\mathrm{t}}$ and $V_{\mathrm{s}}$ are the tip and sample bias, respectively. If the sample surface has a certain area that has locally trapped electric charges, $V_{\mathrm{s}}$ will become the surface potential $V_{\mathrm{q}}$, which is modified by these trapped charges and causes changes in the electrostatic force intensity and the effective spring constant of the cantilever [22].

The resonant frequency, $\omega$ of vibration of the cantilever varies as

$$
\omega=\omega_{0} \sqrt{1-\frac{1}{k} \frac{\mathrm{d} F}{\mathrm{~d} z}}
$$

where $\partial F / \partial z$ is the electrostatic force gradient acting on the EFM probe by these charges. The frequency changes due to the local electrostatic forces could easily be monitored by observing the phase shift of the resulting cantilever vibration.

The phase shift can be expressed as follows:

$$
\Delta \varphi=-\frac{Q}{k} \frac{\partial F}{\partial z}\left(z_{0}\right)=-\frac{Q}{2 k} \frac{\mathrm{d}^{2} C}{\mathrm{~d} z^{2}}\left(V_{\mathrm{EFM}}-V_{\mathrm{q}}\right)^{2}
$$

where $Q$ is the quality factor of AFM probe, $k$ is the spring constant of the cantilever $\left(2.8 \mathrm{~N} \mathrm{~m}^{-1}\right), z$ is the height between the tip and the sample surface, $V_{\mathrm{EFM}}$ and $V_{\mathrm{q}}$ are, respectively, the tip and sample surface potential during charge measurement.

The EFM measurement was conducted on a Veeco Digital Instruments 3100 Dimensions AFM employing a Nanoscope V controller in ambient condition. Charges were injected by using commercial conductive SCM-PIT tip. Its main specifications are: cantilever spring constant, and $\omega_{0}=75 \mathrm{kHz}$. Specifically, the tip is coated with a $\mathrm{Pt} / \mathrm{Ir}$ metal coating.

From (3), a sign change and alteration of $V_{\mathrm{EFM}}$ can induce a bump or a depression in $\Delta \varphi$. Firstly, a p-type silicon (001) substrate with a $3 \mathrm{~nm}$ native oxide layer was charged separately at $-7 \mathrm{~V}$ in figure 2 (a) and $+7 \mathrm{~V}$ in figure 2(b) over $30 \mathrm{~s}$. The $V_{\mathrm{EFM}}$ was set separately at $+2 \mathrm{~V}$ (top region) and $-2 \mathrm{~V}$ (bottom region below). The charged area response is shown in the phase signal. Different signs of $V_{\mathrm{EFM}}$ can change the image colour in the charged region from black to white, which corresponds to a depression or a peak from the phase profiles. By flipping the polarity of the applied voltage the reverse response is found. The uneven surface in the phase image is caused by injected charges that change the sample surface potential.

The original surface phase signal for charge-based memory effects was also measured before charge injection into a sample of Ge NCs on oxidized $\mathrm{Si}$, using two polarities of tip bias ( $\left.V_{\mathrm{EFM}}\right)$ with a magnitude of $\pm 2 \mathrm{~V}$, and height of $50 \mathrm{~nm}$. The resulting phase image is shown in figure 3(b). The average diameter of the Ge NCs is 


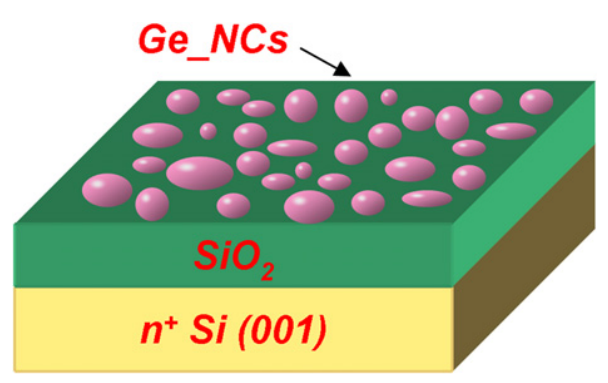

Figure 1. Sample structure with $\mathrm{Ge} \mathrm{NCs}$ on $\mathrm{SiO}_{2}$ layer.

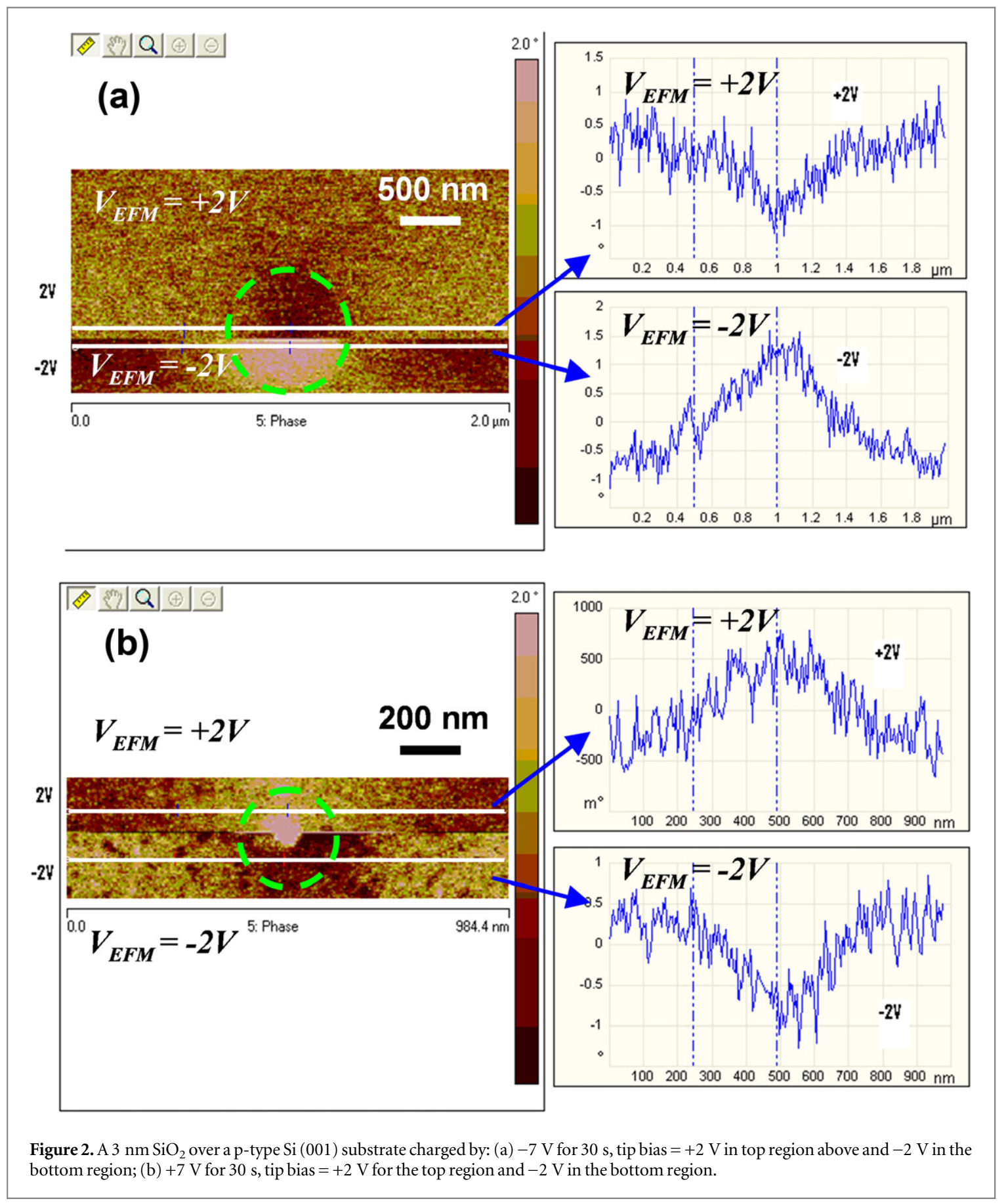




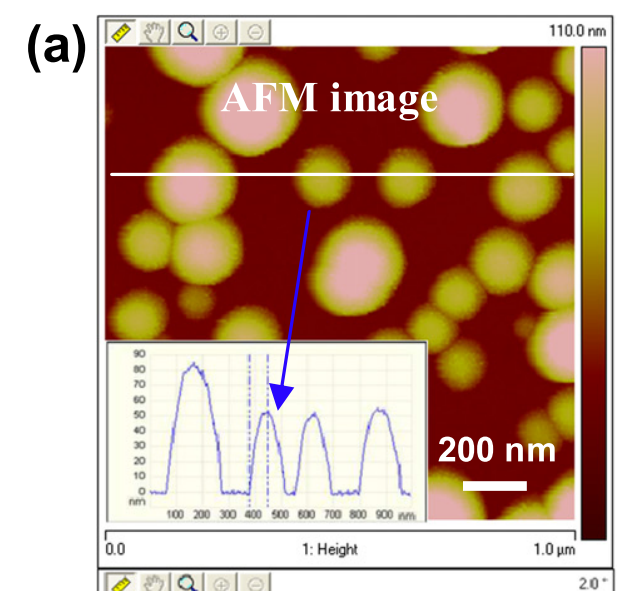

(b)

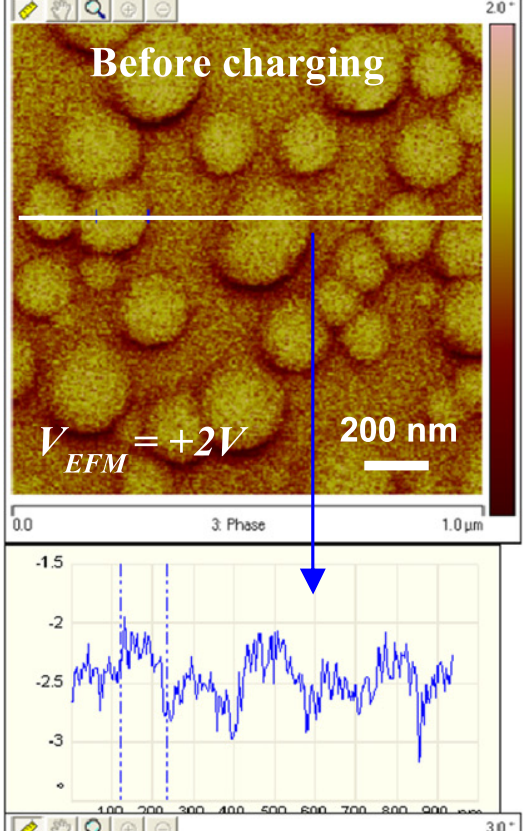

(c)

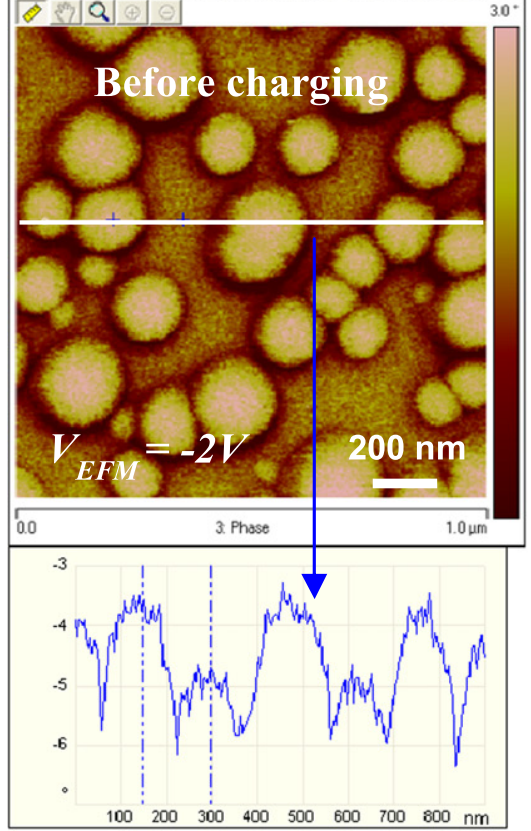

Figure 3. Ge NCs with an average diameter of $150 \mathrm{~nm}$ prior to charging, (a) topography, (b) phase signal images of Ge NCs acquired at $V_{\mathrm{EFM}}=+2 \mathrm{~V}$, (c) Phase signal images acquired at $V_{\mathrm{EFM}}=-2 \mathrm{~V}$. 
$150 \mathrm{~nm}$. The phase signal is found to be higher in the centre of the NC than at the periphery, indicated by its corresponding peak in the phase signal. Additionally, the phase value in (c) was larger than that in (b).

Considering the only change is the polarity and magnitude of $V_{\mathrm{EFM}}$ during these two measurements, it confirms that the Ge NC has quite a weak original positive surface potential $V_{\mathrm{q}}$.

To demonstrate the memory effect based on charging and discharging of individual Ge NCs, an individual Ge NC was identified for charging. For the charging process, the conductive tip was kept at the same position over the isolated NC and subsequently brought closer to the NC surface. At the same time, charges were injected by polarising the tip with a voltage stress of either $+7 \mathrm{~V}$ or $-7 \mathrm{~V}$ for $30 \mathrm{~s}$. The corresponding EFM phase images are shown in figure 4 , where the charged region of the NCs is marked.

From the EFM measurements on a single NC, the charged area can be readily identified. During the charging process, holes $(+7 \mathrm{~V}$, extracted) or electrons $(-7 \mathrm{~V}$, injected) are injected into the isolated Ge $\mathrm{NC}$ where they can form an electron cloud from the phase images. To maximize the resolution of the phase signal a $V_{\mathrm{EFM}}$ with the opposite polarity to $V_{\mathrm{q}}$ is used. The phase shift inside the charged $\mathrm{NC}$ is detected at $0.5^{\circ}\left(V_{\mathrm{EFM}}=+2 \mathrm{~V}\right)$ and $1^{\circ}$ $\left(V_{\mathrm{EFM}}=-2 \mathrm{~V}\right)$ for $+7 \mathrm{~V}$, whereas its value is $-4.5^{\circ}\left(V_{\mathrm{EFM}}=+2 \mathrm{~V}\right)$ and $-2.5^{\circ}\left(V_{\mathrm{EFM}}=-2 \mathrm{~V}\right)$ at the opposite bias of $-7 \mathrm{~V}$. This suggests that it is much easier to inject electrons than holes into Ge NCs.

A particular phenomenon found for charge transfer into Ge NCs is charge localization that is influenced by morphology. From figure 4, the injected charges can be trapped homogenously by the isolated NC and located at the edge. Such Ge NCs became iso-potential and behaved as a conductive material after being charged. It is confirmed that these charges were successfully injected into the isolated Ge NC.

Additionally, from (3), the value of $V_{\mathrm{EFM}}$ can greatly influence the phase shift $\Delta \varphi$. Therefore, comparison between an uncharged and a charged area or these areas charged by different voltages for a fixed $V_{\mathrm{EFM}}$ (tip surface potential) is necessary.

The discharge and retention time of these trapped charges were also evaluated. From figure 5(a), the EFM phase signal is plotted versus time after charging. The phase amplitude in the charged region decreases gradually due to charge dissipation. It is shown that the dominant charge decay mechanism during discharging is the leakage of these trapped charges. The Ge NC is p-type and forms a p-n junction with n-Si, with the associated potential barrier allowing charger to be stored without immediate (Ohmic) discharge/conduction.

Figures $5(\mathrm{~b})$, (c) shows the EFM phase image with different tip bias voltage $V_{\mathrm{EFM}}$ (from $-5 \mathrm{~V}$ to $+4 \mathrm{~V}$ ). The phase intensity of the charged NC follows the magnitude of the tip bias $\left(\left|V_{\mathrm{EFM}}\right|\right)$, where in figure 5(b), this corresponds to the strength of electrostatic force between tip and charged NC. In figure 5(c), phase signal in the centre of the charged region increases with tip bias, and specifically, a negative tip bias alters the indicative phase signal more than a positive bias. This observation confirms that the setup of a $\mathrm{p}-\mathrm{n}$ junction barrier allows trapped charges to be stored with a certain retention time. This is consistent with the experimental evidence that using opposite tip biases for $V_{\mathrm{EFM}}$ and $V_{\mathrm{q}}$ markedly improves resolution in EFM phase image (by at least a factor of 4).

In order to quantitatively characterize the trapped charges, equivalent structures should be used for modelling the interaction between AFM tip and sample surface. The electrostatic force between a conductive tip and a sample surface could be described as [20]:

$$
F_{s}=\frac{1}{2} \frac{\partial C}{\partial z}(\Delta V)^{2}=\frac{1}{2} \frac{\partial C}{\partial z}\left(V_{\mathrm{t}}-V_{\mathrm{s}}\right)^{2}
$$

where $C$ is the effective capacitance between the EFM probe and the sample, $V_{\mathrm{t}}$ and $V_{\mathrm{s}}$ are, respectively, the tip and sample potential. If the sample surface has a certain area that has locally trapped electric charges, $V_{\mathrm{s}}$ will become the surface potential $V_{\mathrm{q}}$ which is modified by these trapped charges and causes changes in the electrostatic force intensity and its gradient. The force gradient changes the effective spring constant of the sensor and, consequently, its resonance frequency $\omega_{\mathrm{r}}$.

$$
\omega_{\mathrm{r}} \approx \omega_{0}\left(1-\frac{1}{2 k} \frac{\partial F}{\partial z}\left(z_{0}\right)\right)
$$

These changes could easily be detected by the phase shift $\Delta \phi$.

$$
\Delta \varphi=-\frac{Q}{k} \frac{\partial F_{\mathrm{s}}}{\partial z}\left(z_{0}\right)=-\frac{Q}{2 k} \frac{\mathrm{d}^{2} C}{\mathrm{~d} z^{2}}\left(V_{\mathrm{EFM}}-V_{\mathrm{q}}\right)^{2}
$$

where $Q$ is the quality factor of AFM probe, $k$ is the spring constant of the cantilever, $z$ is the height of the tip with respect to the surface, $\partial F_{\mathrm{S}} / \partial z$ is the spatial derivative of the electrostatic force acting on the EFM probe by these charges, $V_{\mathrm{EFM}}$ is the tip potential during the EFM measurement.

The AFM probe is composed of three main parts: the cantilever, the tip and the tip apex. During the AFM measurement, these three parts interact with the sample surface and can be modelled simply as a series of flat plane capacitances. The cantilever is modelled as a flat plane, the tip is modelled as a truncated cone [21] and the 
(a)

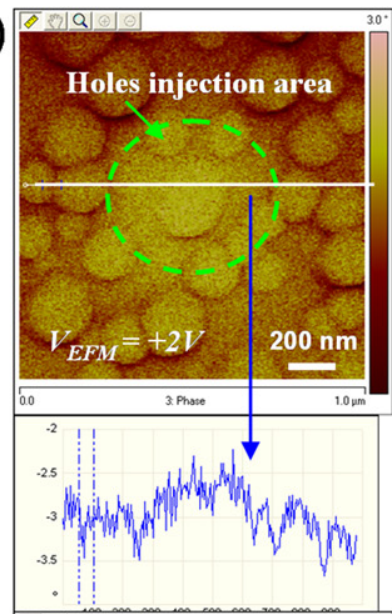

(b)

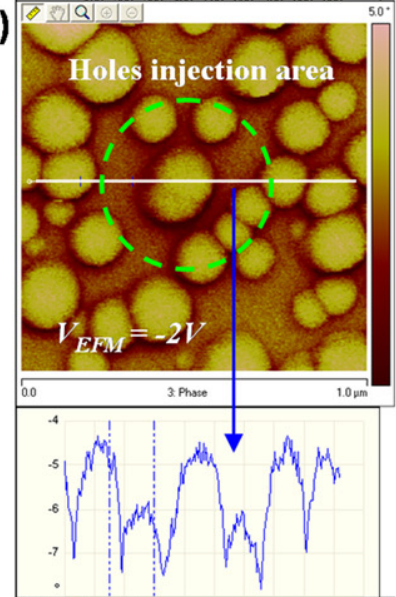

(c)

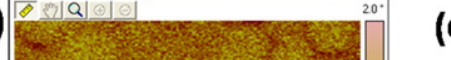

(a)

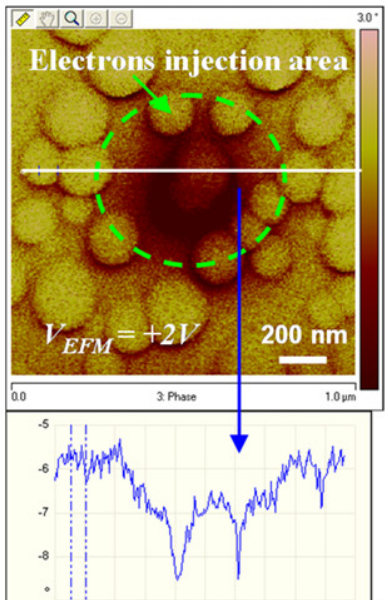

(b)

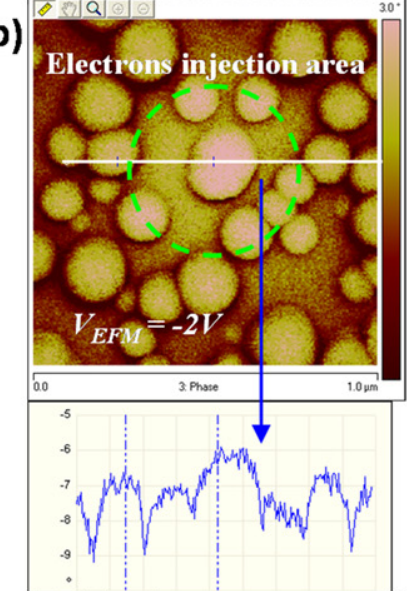

(c)

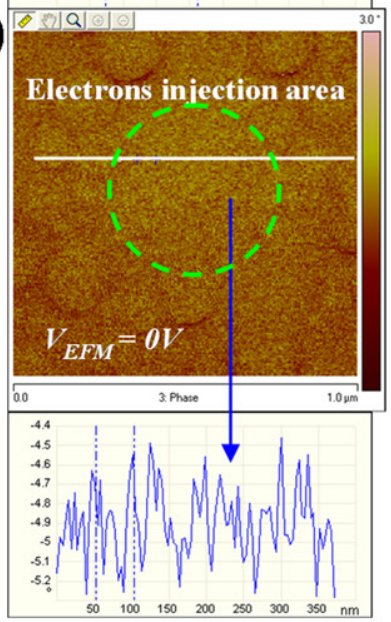

(d)
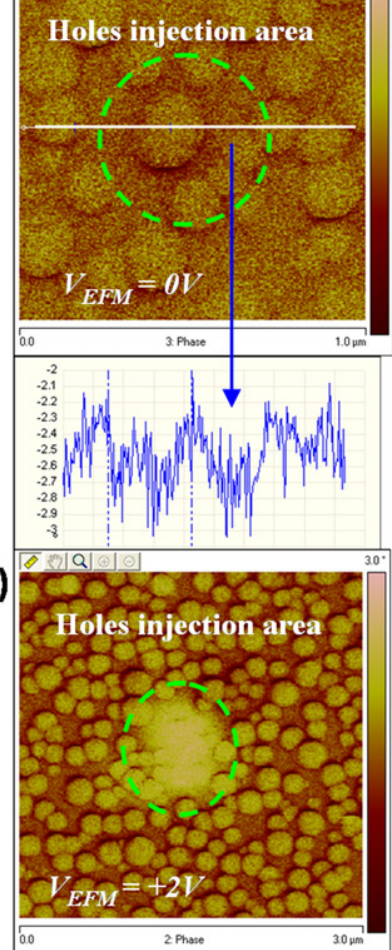

Figure 4. EFM images after charging at $+7 \mathrm{~V} /-7 \mathrm{~V}$ for $30 \mathrm{~s}$. Holes and electrons were separately injected into the isolated Ge $\mathrm{NC}$ as marked. Phase signal images acquired at $V_{\mathrm{EFM}}=(\mathrm{a})+2 \mathrm{~V}$, (b) $-2 \mathrm{~V}$, (c) $0 \mathrm{~V}$, the scan size is $1 \mu \mathrm{m} \times 1 \mu \mathrm{m}$, and (d) $+2 \mathrm{~V}$, the scan size is $3 \mu \mathrm{m} \times 3 \mu \mathrm{m}$. 
(a)

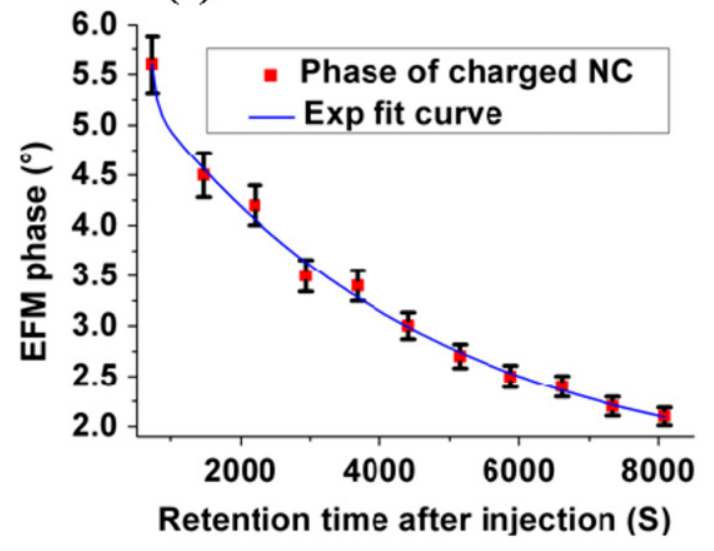

(b)
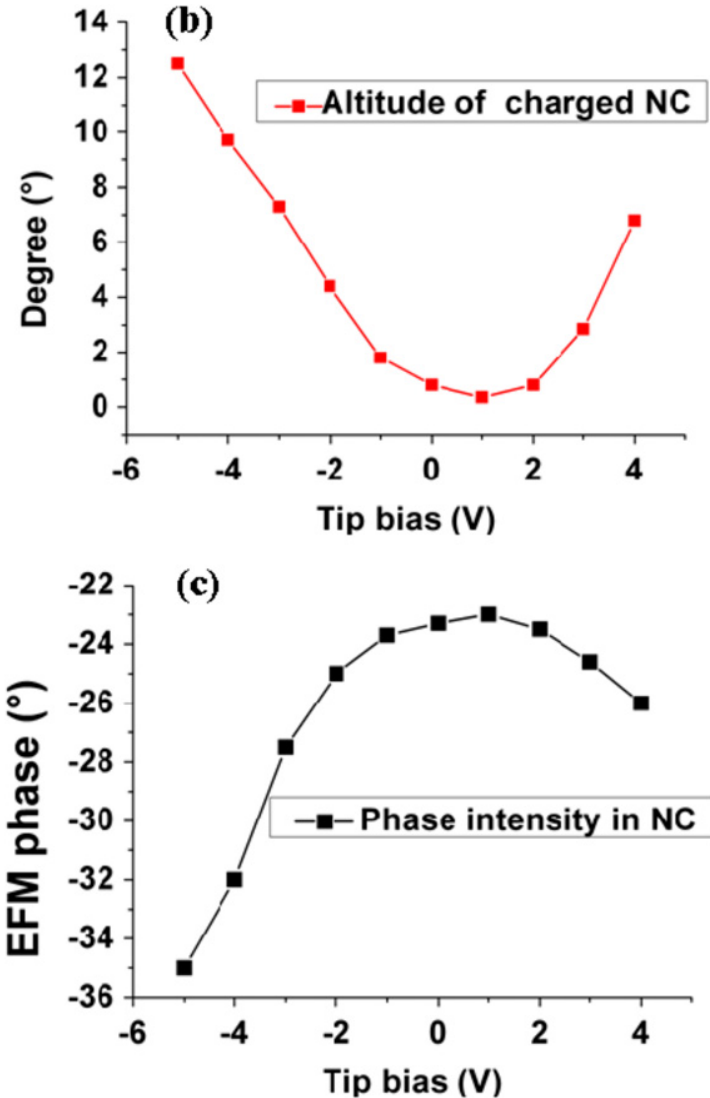

Figure 5. (a) Discharge procedure of trapped charges in the centre of NC, (b) altitude of charged area (c) phase signal in the centre of charged area.

tip apex is modelled as a small sphere or the tip and its apex as a cone. From theoretical studies, the primary contribution to the capacitance variation is given by the tip apex, followed far below by the cone, chip and cantilever [22]. So the truncated cone-plane model is relatively the most accurate approach that considers the tip apex and the tip body.

From the plane-plane capacitance model:

$$
\frac{\partial^{2} C}{\partial z^{2}}=C^{\prime \prime}(z)=2 \varepsilon_{0} \varepsilon_{r} \frac{S}{z^{3}}
$$

The truncated cone-plane model (total tip apex and tip body) gives the second derivative capacitance factor $C_{\text {tcp }}^{\prime \prime}(z)$ :

$$
C_{\mathrm{tcp}}^{\prime \prime}(z) \approx 2 \pi \varepsilon_{0}\left[\frac{R^{2}(2 z+R)}{z^{2}(z+R)^{2}}+k^{2}\left(\frac{1}{z+R}+\frac{R}{\sin \theta(z+R)^{2}}\right)\right] \text { with } \quad k=\frac{1}{\ln \left(\cot \left(\frac{\theta}{2}\right)\right)}
$$

where $R$ is the tip apex radius and $\theta$ is the tip-opening angle.

The equivalent electric circuit of this experiment configuration is given in figure 6 . Where $C_{1}$ is the charged island-substrate capacitance, $C_{2}$ is the charged island-tip capacitance and $C_{3}$ is the substrate-probe capacitance.

Thus, electrostatic force due to these trapped charges can be expressed as:

$$
F=\frac{1}{2}\left(\frac{C_{1} V-q}{C_{1}+C_{2}}\right)^{2} \frac{\mathrm{d} C_{2}}{\mathrm{~d} z}+\frac{1}{2} V^{2} \frac{\mathrm{d} C_{3}}{\mathrm{~d} z}
$$

For SCM-PIT, the tip height is $10-15 \mu \mathrm{m}$ and $\mathrm{R}$ is $20 \mathrm{~nm}$ with a tip-opening angle of $10^{\circ}$. The lift height is always set at $50 \mathrm{~nm}$. The variation of the second derivative capacitance $C^{\prime \prime}(z)$ which varies with the tip-surface distance $\mathrm{z}$ is plotted in figure 7 . We can observe that the effective surface during the tip scan is about $7.1 \times 10^{3} \mathrm{~nm}^{2}$ considering the realistic tip-surface model and a lift height of $50 \mathrm{~nm}$. This effective surface corresponds to a disc area $95 \mathrm{~nm}$ in diameter which is lower than that of the Ge NC. 


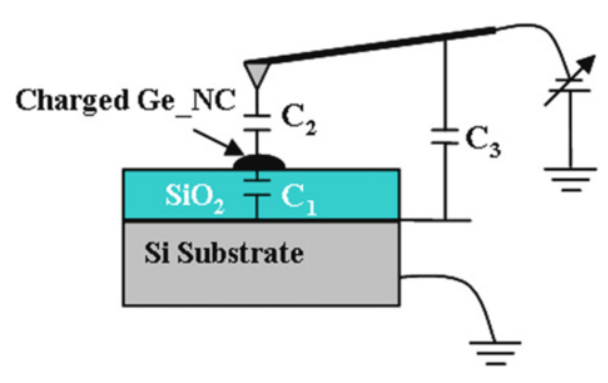

Figure 6. Equivalent circuit.

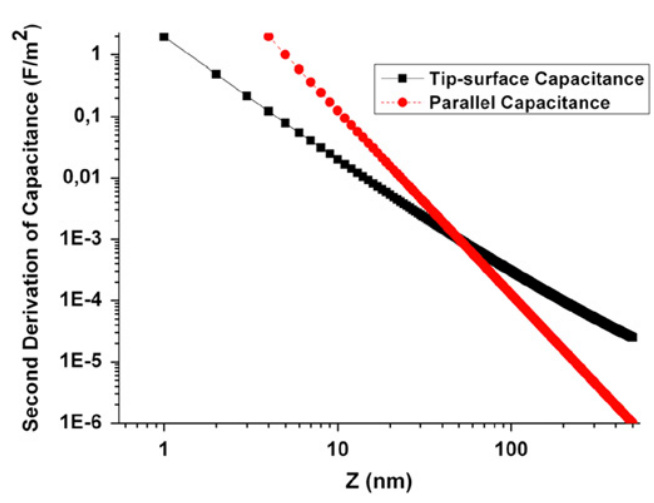

Figure 7. Second derivative capacitance versus tip surface distance.

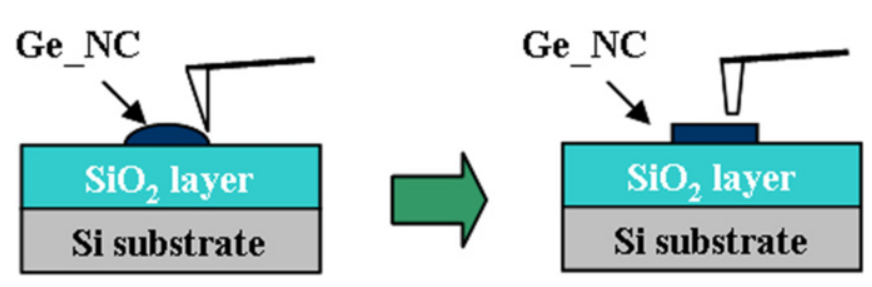

Figure 8. Simplified nano-scale Ge NC model.

The diameter of these isolated Ge NCs is more than $100 \mathrm{~nm}$. However, the SCM-PIT tip has a radius from 20 to $25 \mathrm{~nm}$ which is smaller than the island surface and the effective area is inside it. So these truncated-sphere shape islands could be simplified as rectangular nano structures which have a flat surface interacting with the tip, see figure 8 . This simple model could be used for quantifying trapped charges in oxide layers or embedded conductive nanostructures $[23,24]$.

Using the parallel plate capacitor model, the total charge $q$ inside the trapped area can be deduced as below:

$$
\begin{aligned}
& q=\sqrt{\frac{\Delta \varphi \times k\left(z_{0}+\frac{d_{\mathrm{SiO}_{2}}}{\varepsilon_{\mathrm{SiO}_{2}}}+\frac{d_{\mathrm{Ge}}}{\varepsilon_{\mathrm{Ge}}}\right)^{3} \varepsilon_{0} S}{Q\left(\frac{d_{\mathrm{SiO}_{2}}}{\varepsilon_{\mathrm{SiO}_{2}}}+\frac{d_{\mathrm{Ge}}}{\varepsilon_{\mathrm{Ge}}}\right)^{2}}} \\
& D=\frac{q}{S}
\end{aligned}
$$

where $k$ is the cantilever spring constant, $Q$ is the quality factor of the AFM probe and $S$ is the effective tip area. $D$ is the the charge density in this charged area.

In our experiment, $k$ is $1-5 \mathrm{~N} \mathrm{~m}^{-1}, \mathrm{Q}$ is about 250 , the dielectric constants for $\mathrm{SiO}_{2}$ and $\mathrm{Ge}$ are 3.9 and 16.2, respectively, the charged Ge dot height is about $100 \mathrm{~nm}$ and $S$ is $7.1 \times 10^{3} \mathrm{~nm}^{2}$. Therefore, when the isolated Ge 


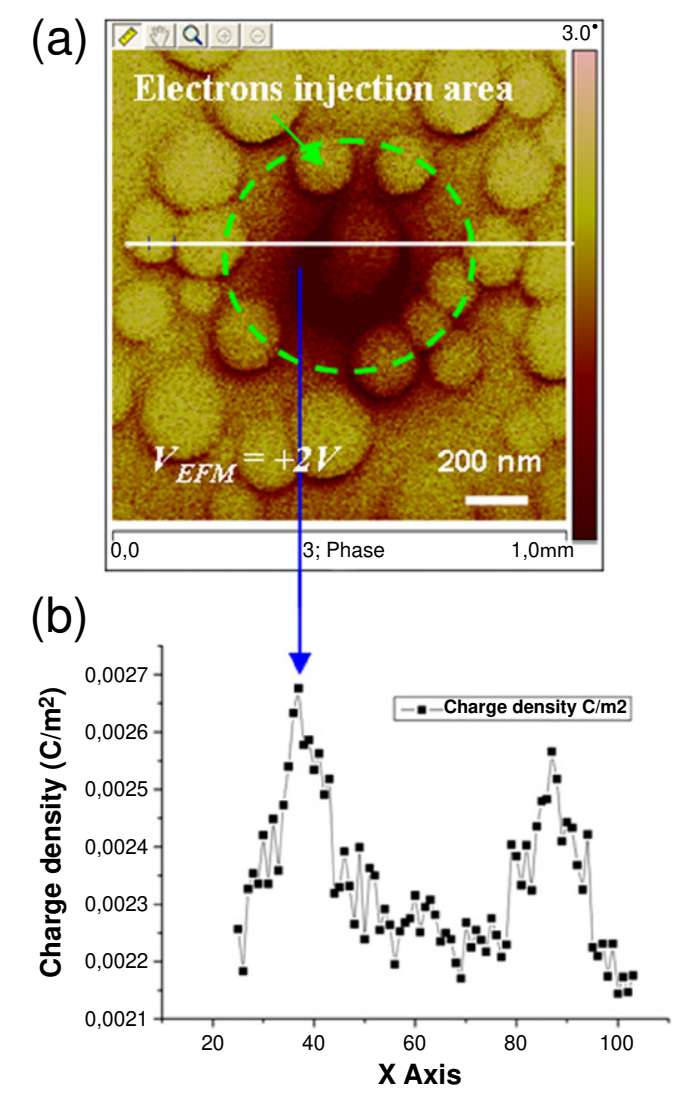

Figure 9. (a) phase image after charge (b) charge density along trapped line.

$\mathrm{NC}$ is charged by $-7 \mathrm{~V}$ for $30 \mathrm{~s}$ (EFM phase image in figures $4(\mathrm{a})$ and (c), electron injection), the injected charge density in the centre of the island is about $7.2 \times 10^{-18} \mathrm{C}$ and that at the edge is about $1.1 \times 10^{-17} \mathrm{C}$. The charge density along the detecting line can be drawn in figure 9 .

From figure 9, the value of charge density was higher at the edge than that inside the charged island. This means that the Ge NC became iso-potential and behaved as a conductive material that caused these trapped charges localize at the edges of its spherical surface.

In the whole charge area, according to our calculation, there are a total of 800 electrons injected to this isolated nano-scale Ge NC.

In summary, EFM two-pass lift mode measurement in ambient condition at room temperature confirmed trapped charges could be stored on single Ge NC on oxidized silicon. Electrons and holes were successfully injected into the Ge NC by a conductive EFM tip. Optimised EFM bias settings for better EFM charge signals have been determined. In order to achieve higher resolution in phase imaging, $V_{\mathrm{EFM}}$ with opposite polarity to $V_{\mathrm{q}}$ is preferred. Such Ge NCs have iso-potential values and behave as a conductive material once charged. The injected charges are also demonstrated to be trapped homogenously by the isolated NC. Trapped electrons inside a $100 \mathrm{~nm}$ NC discharged gradually over $2 \mathrm{~h}$. By varying the magnitude of the tip scan bias, an improvement in the phase signal can be achieved with the use of a negative tip bias, keeping the sample bias positive for this system. By applying a tip bias of $-7 \mathrm{~V}$ for 30 s leads to an injection of about 800 electrons inside an individual Ge NC. This study is of prime importance in developing electronic devices such as memory transistors using Ge NC.

\section{References}

[1] Wildöer J W G, Venema L C, Rinzler A G, Smalley R E and Dekker C 1998 Nature 59391

[2] Odom T W, Huang J L, Kim P and Lieber C M 1998 Nature 62391

[3] Tans S J, Verschueren R M and Dekker C 1998 Nature 49393

[4] Woodside M T and McEuen P L 2002 Science 2961098

[5] Park J Wet al 2002 Nature 417722

[6] Chen W H, Liu C H, Li Q L, Sun Q J, Liu J, Gao X, Sun X H and Wang S D 2014 Nanotechnology 25075201

[7] Graydon O 2013 Nat. Photonics 72

[8] Yang M, Chen T P, Wong J I, Liu Y, Tseng A A and Fung S 2010 J. Nanosci. Nanotechnol. 104517 
[9] Sessolo M, Bolink H J, Brine H, Prima-Garcia H and Tena-Zaera R 2012 J. Mater. Chem. 224916

[10] Chen L-C, Wu Y-C, Lin T-C, Huang J-Y, Hung M-F, Chen J-H and Chang C-Y 2010 IEEE Electron Device Lett. 3112

[11] Binning G, Gerber C and Quate C F 1989 Phys. Rev. Lett. 56930

[12] Lin Z, Bremond G and Bassani F 2011 Nanoscale Res. Lett. 6163

[13] Lin Z, Brunkov P, Bremond G and Bassani F 2010 Appl. Phys. Lett. 97263112

[14] Lemay S G, Janssen J W, van den Hout M, Mooij M, Bronikowski M J, Willis P A, Smalley R E, Kouwenhoven L P and Dekker C 2001 Nature 412617

[15] Odom T W, Huang J-L, Cheung C L and Lieber C M 2000 Science 2901549

[16] Madhavan V, Chen W, Jamneala T, Crommie M F and Wingreen N S 1998 Science 280567

[17] lin T Mé, Deresmes D and Stiévenard D 2002 Appl. Phys. Lett. 815054

[18] Gordon M J and Baron T 2005 Phys. Rev. B 72165420

[19] Marchi F, Dianoux R, Smilde H J H, Murd P, Comin F and Chevrier J 2008 J. Electrostat. 66538

[20] Szkutnik P D, Karmous A, Bassani F, Ronda A, Berbezier I, Gacem K, El Hdiy A and Troyon M 2008 Eur. Phys. J. Appl. Phys. 41 103

[21] Dimension V Scanning Probe Microscope Manual from Vecco Company 279 (2007)

[22] Sarid S 1994 Scanning Force Microscopy (New York: Oxford University Press)

[23] Hudlet S, Saint Jean M, Guthmann C and Berger J 1998 Eur. Phys. J. B 25

[24] Marchi F, Dianoux R, Smilde H J H, Murd P, Comin F and Chevrier J 2008 J. Electrostat. 66538

[25] Schaadt D, Yu E T, Sanar S and Berkowitz A E 1999 Appl. Phys.Lett. 74472

[26] Ng C Y, Chen T P, Lau H W, Liu Y, Tse M S, Tan O K and Lim V S W 2004 Appl. Phys. Lett. 852941 